\title{
EXPRESSION OF IMMUNOGLOBULIN, GRANZYME-B AND PERFORIN AGAINST Ag85A AND Ag85B PROTEINS OF MYCOBACTERIUM TUBERCULOSIS IN BALB/C MICE
}

\section{Fihiruddin $^{1,2}$, Wayan Tunas Artama ${ }^{3,4^{*}}$, Tri Wibawa ${ }^{5}$, Ni Made Mertaniasih ${ }^{6}$}

${ }^{1}$ Doctoral Program, Research Center of Biotechnology, Universitas Gadjah Mada, Yogyakarta. Indonesia;

${ }^{2}$ Department of Medical Laboratory Technology, Politeknik Kesehatan Mataram, Indonesia; ${ }^{3}$ Department of

Biochemistry, Faculty of Veterinary Medicine, Universitas Gadjah Mada, Yogyakarta. Indonesia; ${ }^{4}$ One Health/Ecohealth Resource Center, Universitas Gadjah Mada, Yogyakarta. Indonesia; ${ }^{5}$ Department of Microbiology, Faculty of Medicine, Public Health and Nursing, Universitas Gadjah Mada, Yogyakarta. Indonesia; ${ }^{6}$ Department of

Clinical Microbiology, Faculty of Medicine, Universitas Airlangga, Surabaya. Indonesia

* Corresponding Author's E-mail: artama@ugm.ac.id

\begin{tabular}{|l|}
\hline \multicolumn{1}{|c|}{$\frac{\text { Article History }}{10^{\mathrm{th}}, \text { March, } 2019}$} \\
Received: \\
Revised Received: $21^{\mathrm{st}}$, May, 2019 \\
Accepted: $23^{\mathrm{rd}}$, May, 2019 \\
Published Online: $3^{\text {rd }}$, July, 2019 \\
\hline
\end{tabular}

\section{Abstract}

Background: Ag85 is a protein that may maintain survival of M. tuberculosis in intracellular parts of host cells and is considered as a virulence factor. The expression of Ag85 protein can stimulate proliferation and differentiation of B-cells and T-cells in patients with tuberculosis. This research aimed to determine the ability of Ag85A and Ag85B proteins in activating the response of antibodies, granzyme-B and perforin in Balb/c mice.

Materials and Methods: Twenty-five male Balb/c mice were assigned into five groups. Group I was treated with adjuvant, group II with Bacillus Calmette-Guerin (BCG) vaccine, group III with a combination of BCG and Ag85A, group IV with a combination of BCG and Ag85B and group V with a combination of BCG, Ag85A and Ag85B. Concentrations of immunoglobulin G, granzyme-B and perforin were examined using ELISA and the number of CD8+ T-cells and NK T-cells were checked by flow cytometry.

Results: The highest concentration of immunoglobulin G was found in group V with $62.49 \pm 5.4327 \mathrm{ng} / \mathrm{ml}$. The highest mean number of CD8+ T-cells, NK T-cells, granzyme-B and perforin was found in group IV with 4.32\%, 1.03\%, 35.11 $\pm 1.7789 \mathrm{pg} / \mathrm{ml}$ and $6.19 \pm 0.2235 \mathrm{pg} / \mathrm{ml}$, respectively. The results of One-Way ANOVA test showed that there were significant differences in immunoglobulin responses, with $p<0.05$. The expressions of granzyme-B and perforin were higher in mice treated with combination of BCG and recombinant proteins.

Conclusions: Ag85 protein can be combined with the BCG vaccine to improve protection against M. tuberculosis infection.

Keywords: Ag85A and Ag85B proteins, Immunoglobulin, granzyme B and perforin, TB Vaccine.

List of Abbreviations: Ag85 - Antigen 85, APC - Antigen Presenting Cell, BCG - Bacillus Calmette-Guerin, DOTS - Directly Observed Treatment Short Course, ELISA - Enzyme-Linked Immunosorbent Assay, HIV - Human Immunodeficiency Virus, IL - Interleukin, INF $\gamma$ - Interferon gamma, kDa - Kilodalton, LB - Luria-Bertani Medium, ng/ml - Nanogram/milliliter, MDR Multi Drug Resistant, MIP - Macrophage Inflammatory Protein, MPA - Mycophenolic Acid, NK cells - Natural Killer cell, PHA - Phytohemagglutinin, pg/ml - Picogram/milliliter, Tc - Cytotoxic T cell, WHO - World Health Organization.

\section{Introduction}

Tuberculosis remains a major global health problem because its effective treatment and diagnosis are not established yet (Guggino et al., 2015). Globally, over 10.0 million people that predominately lived in developing countries were infected by tuberculosis in 2017, with a mortality rate of 1.3 million people (WHO, 2016). Bacillus Calmette-Guerin (BCG) vaccination and Directed Observed Treatment Short-course (DOTS) programs are still applied to prevent and treat tuberculosis. Administration of BCG vaccine as a preventive measure for tuberculosis does not provide significant effects and has an efficacy of $0-80 \%$ (Otu, 2013; McBryde et al., 2016; O'Shea et al., 2016). BCG vaccine is effective in miliary tuberculosis or meningitis prevention for children, but does not provide protection in pulmonary tuberculosis for adults with latent tuberculosis and reactivation (Piubelli et al., 2013). Increasing number of Multi Drugs Resistant (MDR) tuberculosis and HIV-related infections cause the cases to be more complex, making the disease increasingly difficult to eliminate. The emergence of MDR tuberculosis and the presence of 
undetected new cases make tuberculosis transmission chain even more uncontrolled (McBryde et al., 2016; O'Shea et al., 2017).

The minimum protection of the BCG vaccine in overcoming tuberculosis transmission and epidemics has encouraged continuous efforts to find new candidate vaccines (Dietrich et al., 2014). Development of better diagnostic tools and the use of new vaccines are two main pillars to reduce MDR TB cases (Horwitz et al., 2000). The discovery of new, more effective vaccine candidates, including the vaccine sub-unit, is an effort that has been done to control and eliminate tuberculosis (Jiang et al., 2013). A main fraction of the excretory protein of M. tuberculosis and M. bovis BCG culture filtrate is the Ag85 complex. Secreted antigen 85 (Ag85) protein is present on the cell wall surface and needed for the survival of M. tuberculosis in macrophages and is considered a virulence factor. Three members of Ag85 protein with molecular weights of 30-32 kDa: Ag85A, Ag85B and Ag85C that are encoded by three paralogous genes: $f b p A, f b p B$ and $f b p C$, are located in different regions of the bacterial genome (Kuo et al., 2013).

Ag85 is known as a protein that can maintain survival of M. tuberculosis in intracellular parts of host cells and helps the bacteria defend themselves against the immune system while facilitating the formation of tubercles (Launois et al., 2011; Piubelli et al., 2013 Zarif et al., 2013). Expressions of protein Ag85 by $f b p A$ and $f b p B$ genes can stimulate proliferation and differentiation of B cells and T- cells. T cells can be differentiated into Th1, Th2, Treg and Tc cells. B cells are activated by Ag85 antigens which will stimulate the production of $\operatorname{IgM}$ or IgG antibodies against M. tuberculosis. Ag85 protein can induce a strong immune response with significant secretion of cytokines by Th1 cells, including Interleukin-2 (IL-2) and Interferon-gamma (IFN$\gamma$ ) that are required to regulate the number of immune cells and induce cytotoxic cells (Wang et al., 2010). T-cells will activate macrophage cells, NK cells, DC cells and Tc cells through cytokines produced to destroy M. tuberculosis on a cellular basis (Jiang et al., 2015; Rizzi et al., 2012; Metcalfe et al., 2016).

Ag85 protein of $M$. tuberculosis can induce macrophages and dendritic cells to produce Macrophage Inflammatory Protein (MIP) such as MIP-1 $\beta$ (CCL4) and secretes IL-15. MIP-1 $\beta$ and IL-15 can increase Tc cell and NK cell activation and proliferation. Activated Tc cells and NK cells can kill cells infected by $M$. tuberculosis by producing chemical mediators, such as granzyme-B and perforin (Wang et al., 2010; Kuo et al., 2013; Silva et al., 2013). Ag85A (32 kDa) and Ag85B (30 kDa) proteins are potential candidates for tuberculosis vaccines, because they are the main proteins secreted by M. tuberculosis and show strong immunogenic properties (Yuk and Jo., 2014). This study aimed to determine the ability of Ag85A and Ag85B proteins in activating the response of antibodies, granzyme-B and perforin in Balb/c mice.

\section{Materials and Methods Animal models}

In this research, twenty-five ( $\mathrm{n}=25$ ) male Balb/c mice (8-10 weeks old, 20-30 g) were obtained and kept at the animal experimental laboratory of LPPT Universitas Gadjah Mada. Ethical clearance for use of animals in research was obtained from the Ethical Clearance Commission of the Faculty of Veterinary Medicine, Universitas Gadjah Mada (Approval Number: 0021/ECFKH/Eks/2018). The mice were kept in standard conditions as demanded by humane protocol and each cage contained five Balb/c mice.

\section{Preparation of Ag85 recombinant protein}

Ag85A and Ag85B genes were inserted into pET SUMO plasmid (InvitrogenTM ChampionTM pET SUMO Cat Protein Expression System No. K300-01) and were transformed into E. coli BL21 (DE3). Ag85 recombinant genes were expressed in liquid LB medium containing kanamycin $(50 \mu \mathrm{g} / \mathrm{ml})$, and induced with $1 \mathrm{mM}$ isopropyl-beta-D-thiogalactopyranoside (IPTG). Ag85A and Ag85B recombinant proteins were then purified by Ni-NTA purification system kit (InvitrogenTM NovexTM Cat No.K950-01). The purified proteins were cleaved, using protease to separate the SUMO taq protein in SUMO and Ag85 recombinant proteins, and stored at $-20^{\circ} \mathrm{C}$.

\section{Immunization of Balb/c mice}

Immunogenicity of Ag85 recombinant proteins of $M$. tuberculosis was measured in Balb/c mice that had been immunized by Ag85A and Ag85B proteins. The expressions of the Balb/c mice antibodies and cellular immune responses were analyzed. The animals were assigned into five groups, which consisted of five Balb/c mice per group. Group I was treated with adjuvant, group II with BCG vaccine (Bio Farma Bandung), group III with a combination of BCG and Ag85A, group IV with a combination of BCG and Ag85B, and group V with a combination of BCG, Ag85A and Ag85B. Initially, Balb/c mice were vaccinated by using $0.2 \mathrm{ml}$ of recombinant proteins containing $10 \mu \mathrm{g}$ of Ag85A and Ag85B, which was supplemented with Complete Freund Adjuvant (1:1) intraperitoneally in groups III, IV and V. Booster was done every two weeks for three times with $5 \mu \mathrm{g}$ of an antigen supplemented with Incomplete Freund Adjuvant. The same treatment was also given to the control group (Chen et al., 2006). Animals were euthanized based on Institutional Animal Care and Use Committee (IACUC) by using the Cervical Dislocation method. Lymphocytes (splenocytes) from the lymph of Balb/c mice were then isolated for further examination of CD8+ T-cells and NK cells using flow cytometry methods.

\section{Serum collection and ELISA assay}

Blood samples of Balb/c mice were obtained through the sinus orbital bleeding. Previously, mice were anesthetized with $40 \mathrm{mg} / \mathrm{kg} \mathrm{BW}$ ketamine (IACUC standard). Blood samples were kept in $1.5 \mathrm{ml}$ microcentrifuge tube, and subjected to centrifuge at 6,000 rpm for 10 minutes. The serum obtained was examined for immunoglobulins, granzyme-B and perforin. Immunoglobulin examination was conducted by using anti-mouse IgG $(\mathrm{H}+\mathrm{L})$ AP secondary antibody (Invitrogen (2010). Cat 
No.A16069). Perforin was examined by using mouse perforin/pore-forming ELISA kit protein (Cat Bioassay technology - No. E0999Mo); while granzyme-B was examined by using mouse granzyme-B ELISA kit (Affymetrix ebioscience - BMS 6029).

\section{Isolation of splenocytes in Balb/c mice and flow cytometry assay}

Spleens of Balb/c mice were aseptically isolated, and transferred into sterile petri dishes containing RPMI 1640 culture medium. Cells were removed from the spleen by RPMI 1640 medium spraying using a syringe repeatedly. The cells suspension was moved into a-15 ml conical tube and centrifuged at 5,000 rpm for 5 minutes. The erythrocytes were lyzed with $1 \mathrm{ml}$ ammonium chloride $\left(\mathrm{NH}_{4} \mathrm{Cl}\right)$ solution for 3 minutes, and then centrifuged at 5,000 rpm for 5 minutes. Lymphocytes were dissolved in RPMI 1640 medium, and centrifuged at 5,000 rpm for 5 minutes. The pellets were dissolved in $100 \mu 1$ PBS, and transferred 50

$\mu 1$ solution to a new tube and mixed with $5 \mu 1$ of anti-mouse CD8+ FITC/anti-mouse CD49b FITC monoclonal antibody. The solution was incubated for 30 minutes at room temperature in a dark condition. It was then subjected for flow cytometry assay (FACS Calibur, Beckton Dickinson, San Jose, CA) and analyzed by using CELL QUEST software (Beckton Dickinson).

\section{Statistical \\ Analysis}

All values were presented as means \pm standard deviations. Numerical data were analyzed using One-way ANOVA test for normally distributed and homogeneous data. Data analysis was performed by using SPSS 16.0 software with a confidence level of $95 \%$. Differences were considered as statistically significant at $p<0.05$ values.

\section{Results}

Recombinant Ag85A and Ag85B proteins were expressed in the BL21 (DE3) competent E. coli expression system. Figure 1 shows that $\mathrm{Ag} 85 \mathrm{~A}$ and $\mathrm{Ag} 85 \mathrm{~B}$ have $36 \mathrm{kDa}$ and $34 \mathrm{kDa}$ molecular weights, respectively. This confirmed that the recombinant $\mathrm{Ag} 85 \mathrm{~A}$ and $\mathrm{Ag} 85 \mathrm{~B}$ were appropriate for further in vivo analysis.
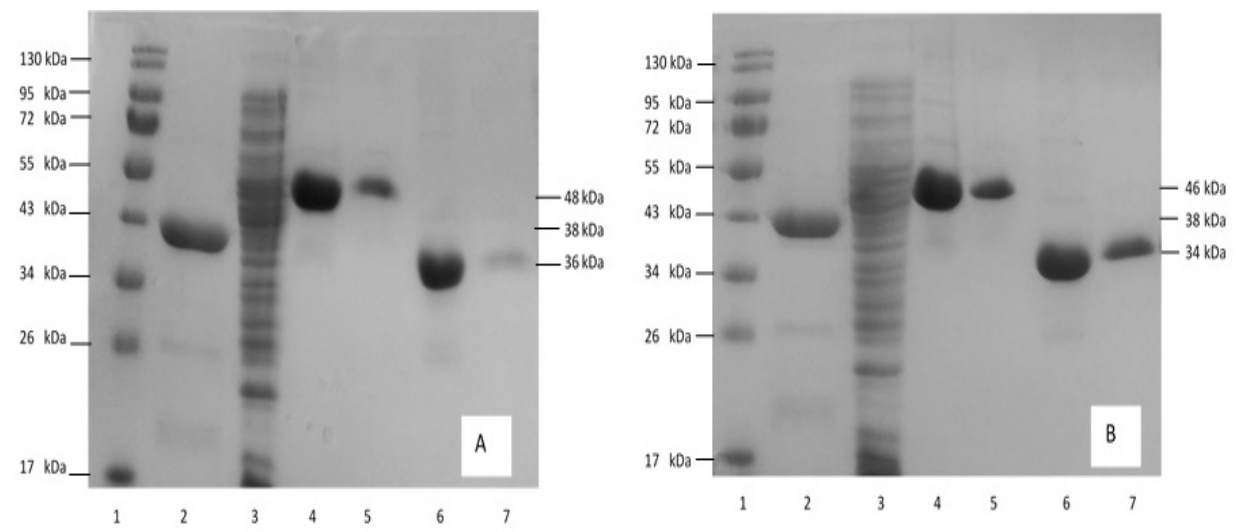

Figure 1: SDS-PAGE of Ag85 protein expressed by BL21 (DE3) competent $E$. coli cells grown on LB broth media induced with $1 \mathrm{mM}$ IPTG. Line 1: protein loading, line 2: pET SUMO/CAT proteins (positive control), line 3: BL21 (DE3) competent E. coli cells without recombinant plasmids, line 4 and 5: Ag85A protein, line 6 and 7: Ag85A protein was cleaved by protease enzymes (A) and Line 1: protein loading, line 2: pET SUMO/CAT proteins, line 3: BL21 (DE3) competent E. coli cells without recombinant plasmids, line 4 and 5: Protein Ag85B, line 6 and 7: Ag85B protein was cleaved by protease enzyme.

Intra-peritoneal administrations of recombinant $\mathrm{Ag} 85 \mathrm{~A}$ and $\mathrm{Ag} 85 \mathrm{~B}$ proteins induced the antibody production against $\mathrm{Ag} 85 \mathrm{~A}$ and Ag85B in Balb/c mice (Figure 2). The results showed that the antibody expression was significantly higher in mice treated with combination of BCG and Ag85A or Ag85B and both recombinant proteins compared to the untreated and BCG treated mice ( $p=0.001)$. Recombinant Ag85A and Ag85B proteins clearly have immunogenicity effect in Balb/c mice. 


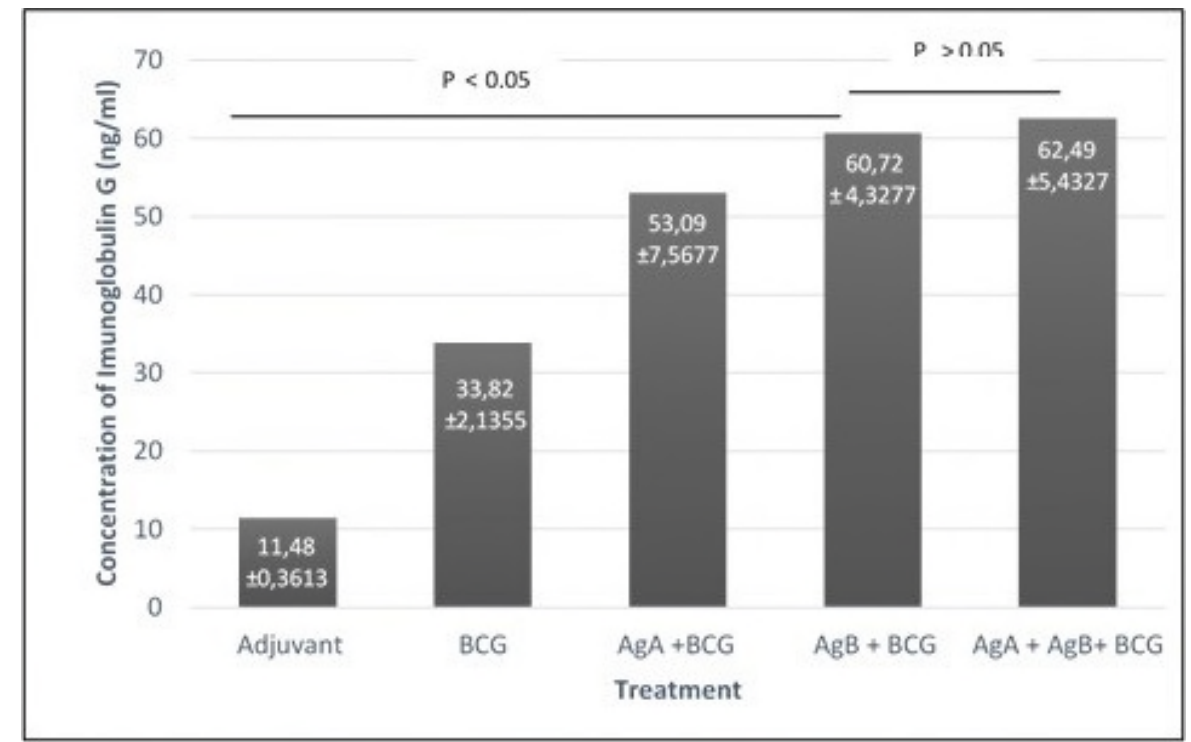

Figure 2: Concentration of immunoglobulin $\mathrm{G}$ in serum of Balb/c mice using ELISA test. Each treatment of groups was induced by adjuvant, BCG, BCG combined with Ag85A protein, BCG combined with Ag85B protein and BCG combined with both $\mathrm{Ag} 85 \mathrm{~A}$ and $\mathrm{Ag} 85 \mathrm{~B}$ proteins.
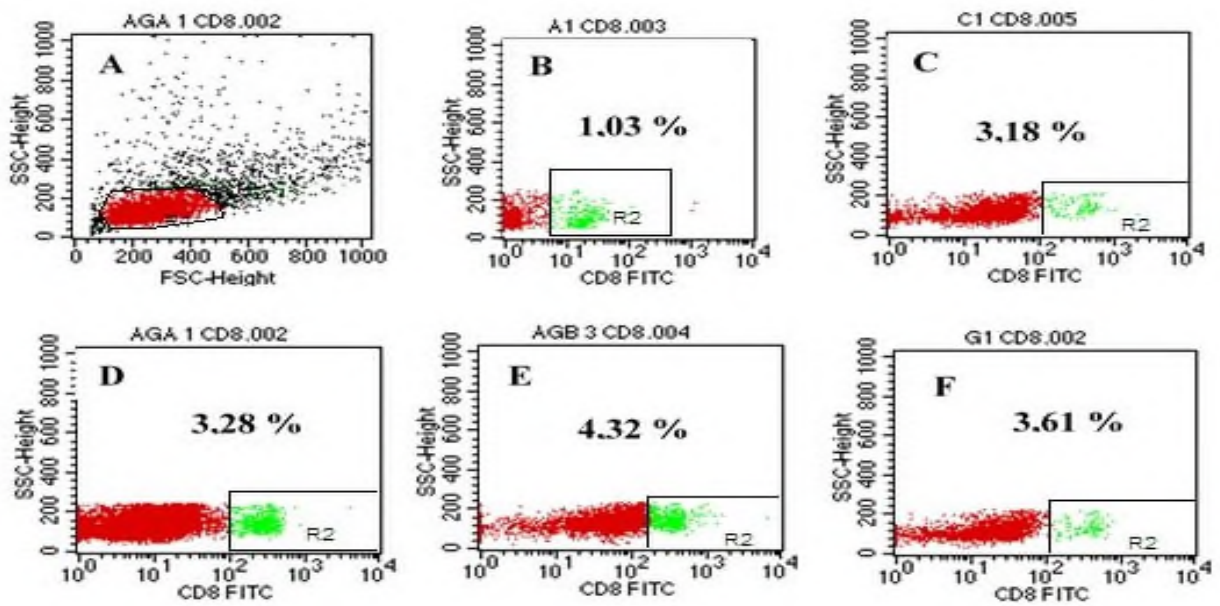

Figure 3: Flow cytometry of CD8+ T-cells. The number of CD8+ T-cells in spleen of Balb/c mice detected by using anti-mouse CD8+ FITC in various treatment groups. Control cells without monoclonal antibodies (A). The number of CD8+ T-cells in Balb/c mice induced with adjuvant (B), induced with BCG (C), induced with a combination of BCG and Ag85A (D), induced with a combination of BCG and Ag85B (E), and induced with a combination of BCG and both of Ag85A and Ag85B (F).
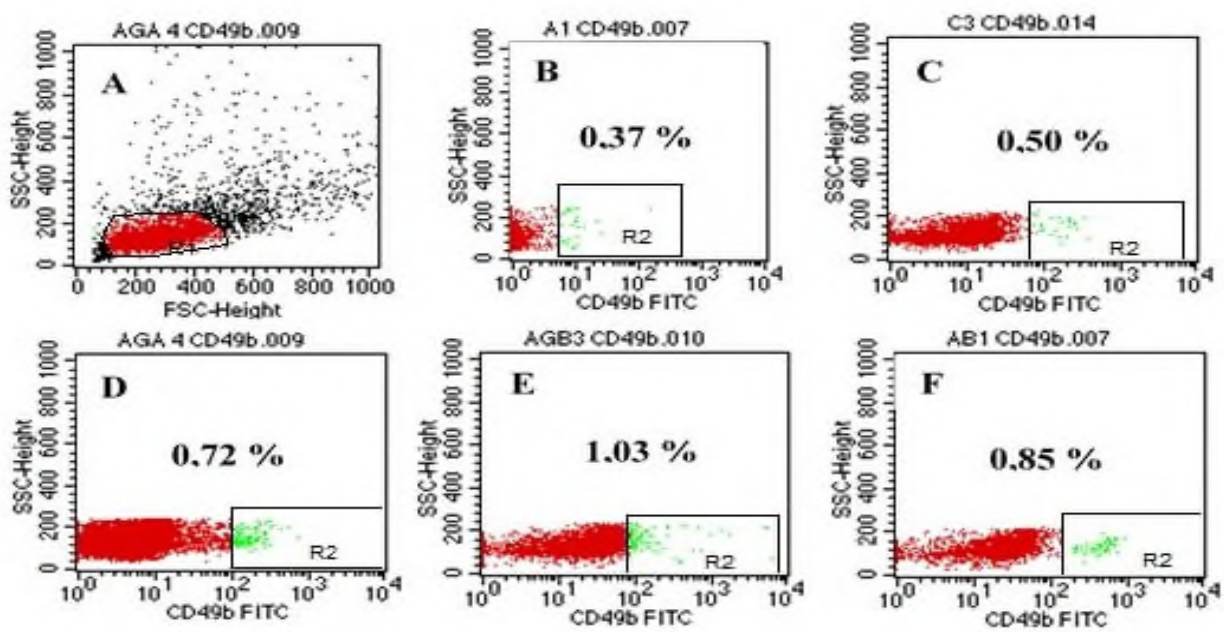

Figure 4: Flow cytometry of NK cells. The number of NK cells in spleen of Balb/c mice were detected by using anti-mouse 49b FITC in various treatment groups. Control cells without monoclonal antibodies (A). The number of NK cells in Balb/c mice induced with adjuvant (B), induced with BCG (C), induced with a combination of BCG and Ag85A (D), induced by a combination of BCG and Ag85B (E), and induced by a combination of BCG and both of Ag85A and Ag85B (F) 
The mean number of CD8+ T-cells in Balb/c mice induced by adjuvant, BCG, BCG combined with Ag85A, BCG combined with Ag85B, and BCG combined with both of Ag85 A and Ag85B were 1.03\%, 3.18\%, 3.28\%, 4.32\% and 3.61\%, respectively (Figure 3). The mean numbers of NK cells were $0.37 \%, 0.5 \%, 0.72 \%, 1.03 \%$ and $0.85 \%$, respectively (Figure 4 ). The scatter plot indicated that, the highest percentage of CD8+ T-cells and NK cells number were observed in the combination of BCG and Ag85B groups. The One-Way ANOVA test showed that the mean number of CD8+ T-cells and NK cells were significantly higher in mice treated with combination of BCG and Ag85A or Ag85B and both recombinant proteins compared to the untreated and BCG treated mice $(p=0.001$ and $p=0.035)$, respectively. Whereas, the mean number of CD8+ T-cells and NK cells were not significantly different in mice treated with combination of BCG and Ag85A compared to the combination BCG and Ag85B or both recombinant proteins, with a value of $p>0.05(p=0.14$ and $p=0.11)$, respectively.
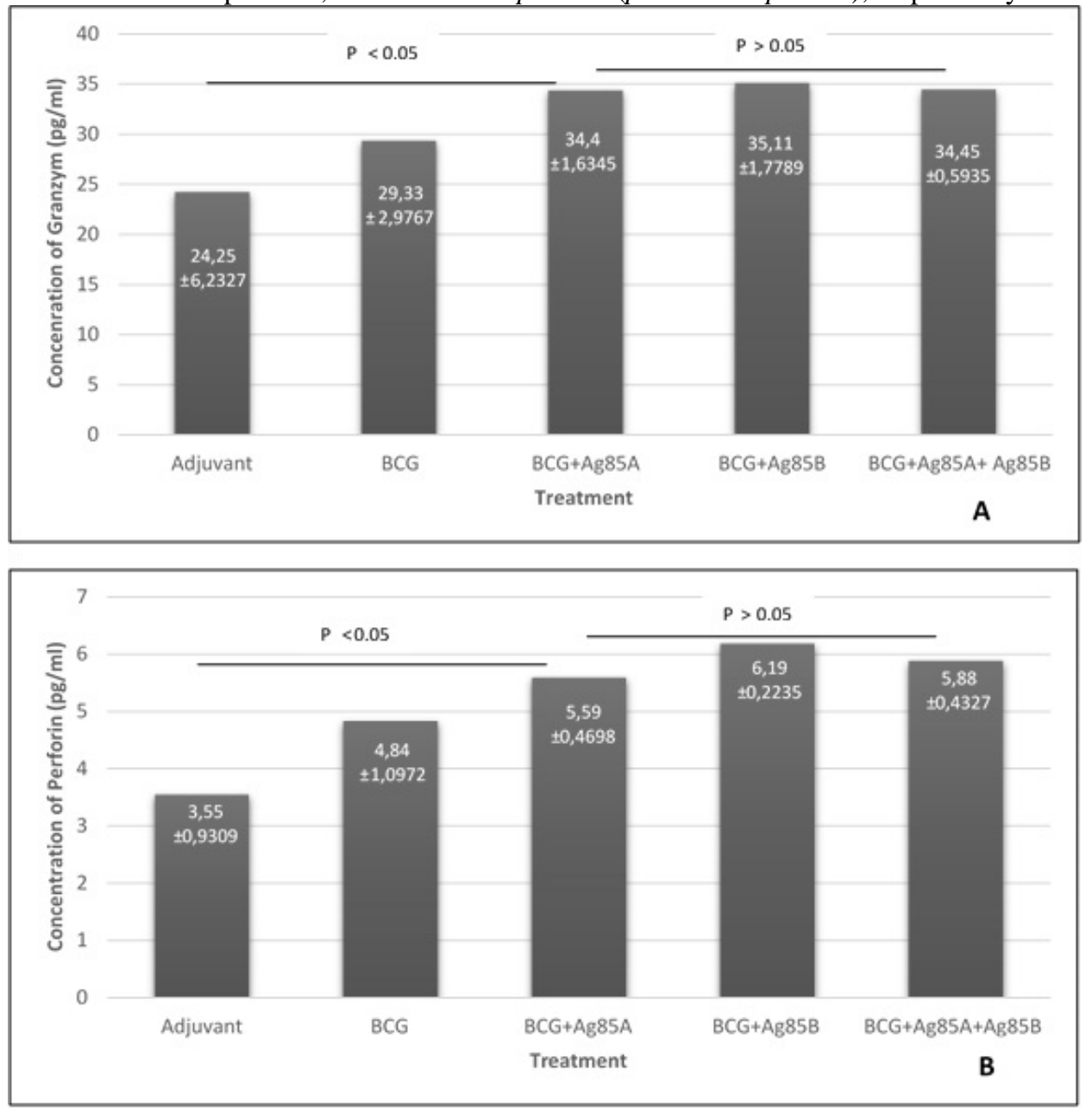

Figure 5: Results of ELISA test of perforin (A) and granzyme-B (B) in serum of Balb/c mice induced by various treatments separately. Each treatment of groups was induced by adjuvant, BCG, BCG combined with Ag85A protein, BCG combined with Ag85B protein and BCG combined with Ag85A and Ag85B proteins.

Results of ELISA test of granzyme-B and perforin on each of the groups I, II, III, IV and IV were $24.25 \mathrm{pg} / \mathrm{ml}$ and 3.55 $\mathrm{pg} / \mathrm{ml}, 29.33 \mathrm{pg} / \mathrm{ml}$ and $4.84 \mathrm{pg} / \mathrm{ml}, 34.4 \mathrm{pg} / \mathrm{ml}$ and $5.59 \mathrm{pg} / \mathrm{ml}, 35.11 \mathrm{pg} / \mathrm{ml}$ and $6.19 \mathrm{pg} / \mathrm{ml}$ as well as $34.45 \mathrm{pg} / \mathrm{ml}$ and $5.88 \mathrm{pg} / \mathrm{ml}$, respectively.

These results indicated that the highest concentrations of granzyme-B and perforin were found in the group induced with BCG and Ag85B. The concentrations of granzyme-B and perforin were significantly higher in mice treated with combination BCG and Ag85A or Ag85B and both combination proteins compared to untreated mice. Statistical tests showed no significant differences in the levels of granzyme-B and perforin in mice treated with combination BCG and Ag85A or Ag85B and both recombinant proteins compare to BCG treated mice, with a value of $p>0,05(p=0, .06$ and $p=0.10)$, respectively.

\section{Discussion}

Various efforts have been made to control and prevent morbidity and mortality of patients infected with $M$. tuberculosis. Discovery of a new vaccine candidate with high antigenicity in improving the immune response was one of the efforts that continues to be developed (Aghababa et al., 2011). Several M. tuberculosis excretory proteins play important roles in the pathogenesis of the disease and act as immunostimulants that directly interact with the host's immune system. Ag85A and Ag85B proteins of $M$. tuberculosis proteins are some of the candidates that could be used for the development of TB vaccines. Recombinant Ag85 proteins of M. tuberculosis have been shown as a promising approach (Zvi et al., 2008; Kuo et al., 2013). In this study, our Ag85A and Ag85B recombinant proteins expressed in BL21 (DE3) competent E. coli cells showed functional 
properties to induce immune response in Balb/c mice.

The results of the immune response tests of $\mathrm{Ag} 85 \mathrm{~A}$ and $\mathrm{Ag} 85 \mathrm{~B}$ proteins on the experimental animals used showed that a significant increase in immune response $(p<0.05)$ after Balb/c mice were administered a combination of Ag85 recombinant protein compared to the BCG vaccinated only group. The combination of BCG and Ag85B had the highest antigenic activity in stimulating antibody formation in Balb/c mice. Ag85B proteins with a molecular weight of $34 \mathrm{kDa}$ were the dominant antigen and responsible for almost $25 \%$ of the total extracellular protein. Vaccinations of the experimental animals that were induced with Ag85B gave significant protective immunity to the exposure against $M$. tuberculosis compared to the conventional $M$. bovis BCG vaccine. The study conducted by Li et al. (2015) in female ICR mice demonstrated an enhanced T- cell response and increased production of cytokines, including interferon- $\gamma$, tumor necrosis factor- $\alpha$ and interleukin- 2 in mice treated with a combination of BCG and recombinant adenoviral vector (Ad5-CEAB) co-expressing Ag85 and culture filtrate protein, compared to treatment with BCG alone. In addition, the subjects of this study demonstrated to have a humoral response with increased concentrations of secretory immunoglobulin $\mathrm{A}(\mathrm{sIg} \mathrm{A})$ and serum immunoglobulin $\mathrm{G}(\mathrm{IgG})$ in mice treated with a combination of $\mathrm{BCG}$ and recombinant adenoviral vector (Ad5-CEAB), compared to treatment with BCG alone. The study conducted by Tanghe et al. (2001) revealed that protein boosting (Ag85A and Ag85B) increased the protective efficacy of a tuberculosis DNA vaccine encoding Ag85 against M. tuberculosis H37Rv challenge infection in C57BL/6 mice. This result may indicate that Ag85 is a good candidate for vaccine development.

The Antigen Ag85 complex (Ag85A and Ag85B) was capable of stimulating proliferation of NK-cells and CD8+ Tcells. The results of this study revealed that there were differences in the number of CD8+ T-cells and NK-cells in each group when it was compared with Balb/c mice without Ag85 protein induction, although it was not statistically significant ( $\mathrm{p}>0.05)$. NK-cells and CD8 + T-cells are components of cells crucial for cellular immunity, due to their actions as first defense systems in encountering pathogenic viruses and bacteria. Vaccination with DNA plasmid encoding Ag85 and modified vaccination virus Ankara strain that expressed Ag85 protein could induce CD4+ T-cell and CD8+ T-cell responses in Balb/c mice. Ag85 protein contains epitopes that could be recognized by the Major Histocompatibility Complex (MHC) classes I and II (Huygen, 2014). Administration of BCG vaccine (that had plasmid inserted Ag85B) in sheep induced high levels of IL-4 and IL-17 in peripheral blood mononuclear cells compared to BCG vaccine (Rizzi, 2012).

Recognition of M. tuberculosis antigen by macrophage cells triggered the activation and production of cytokines. Several of the cytokines produced in tuberculosis infection process include INF- $\gamma$, IL-4, IL-12, IL-6, IL-8 and IL-10. These cytokines can trigger humoral and cellular immune responses (Van-Crevel et al., 2002; Tambunan et al., 2018). IL-2 produced by macrophage cells that are activated by Ag85 protein, which is able to stimulate T-cell proliferation, was also identified as the cytokine responsible for increasing T-cell and B-cells differentiation. Interleukin-2 Beta Receptor (IL-2R) cells are known to be bound to IL-15 produced by various types of cells and tissues, that generally have biological functions with IL-2, such as T-cells, B-cells, NK-cells, monocyte cells, dendritic epidermal cells, timocytes and granulocytes (Endharti, 2007). Macrophage and dendritic cells activated by Ag85 recombinant protein also produce MIP-1 $\beta$ (CCL4) and secrete IL-15. MIP-1 $\beta$ and IL-15 could increase activation and proliferation of T-cells and NK-cells. Activated CD8+ T-cells and NK-cells could lyse infected cells by producing cytotoxic molecules in the form of granzyme-B and perforin (Wang et al., 2009; Kuo et al., 2013; Silva et al., 2013).

Our study showed that BCG combined with Ag85 recombinant protein could not induce CD8+ T-cells and NK-cells to express granzyme-B and perforin significantly in all treatment groups. However, an in vitro study conducted by Lu et al. (2015) revealed that NK-cells cultured with $M$. tuberculosis bacteria might express perforin and granulysin to kill bacteria. The study conducted by Huang et al. (2006) also indicated that Jurkat T-cells (immortal cells line) activated with PHA and PMA in vitro, produced increased granzyme-B expression controlled through the NF-kB binding site. Ag85 protein was hypothetically expected to increase the expression of granzyme-B and perforin in latent TB patients (LTBI) to destroy any infected cells. Mice with disorders of granzyme-B and perforin excretions might experience other disorders, such as a disorder of eliminating viruses or pathogen bacteria. Granzyme-B and perforin are two molecules triggering cytotoxicity in either NK-cells or CD8+ T-cells (Huang et al., 2006). Both cells result in cytotoxic molecules, suggesting that cytokines could kill M. tuberculosis. Additionally, they could recognize antigens represented by MHC class I existing on the surface of APC cells (Axelsson-Robertson et al., 2015).

\section{Conclusion}

Ag85A and Ag85B proteins act as immunogenic agents, that could increase immunoglobulin response and the number of CD8+ T-cells and NK-cells in Balb/c mice induced by BCG combined with recombinant proteins. However, they could not activate CD8+ T-cells and NK-cells to express granzyme-B and perforin significantly.

Conflict of Interest: The authors declare that there is no conflict of interest in this study.

\section{Acknowledgement}

The authors would like to thank the staff of One Health/Ecohealt Resource Center, Gadjah Mada University (OH/EH RC-UGM). This project was supported by PDUPT Ristek Dikti of Republic of Indonesia (NO: 177/UNI/DITLIT/DITLIT/TL/2018).

\section{References}

1. Aghababa, H., Mobarez, A.M., Behmanesh, M., Khoramabadi, N., and Mobarhan, M. (2011). Production and purification of mycolyl transferase B of Mycobacterium tuberculosis. Tanaffos, 10(4):23-30. 
2. Axelsson-Robertson, R., Rao, M., Loxton, A.G., Walzl, G., Bates, M., Zumla, A., and Maeurer, M. (2015). Frequency of Mycobacterium tuberculosis-spesific CD8+T-cell in the course of anti-tuberculosis treatment. International Journal of Infectious Diseases, 32: 23-29.

3. Chen, M., Gan, H., and Remold, H.G. (2006). A mechanism of virulence: virulent Mycobacterium tuberculosis stain H37Rv, but not attenuated H37Ra, causes significant mitochondrial inner membrane disruption in macrophages leading to necrosis. Journal of Immunology, 176(6):3707-3716.

4. Dietrich, J., Aagard, C., Leah, R., Olsen, A.W., Stryhn, A., Doherty, T.M., and Andersen, P. (2014). Vaccine efficacy. Journal of Immunology, 174:6332-6339.

5. Endharti, A.T. (2007). Spontaneous activation of T cell Lymphocytes in mice lacking interleukin-2 receptor. Journal Kedokteran Brawijaya, 13(1):40-46.

6. Guggino, G., Orlando, V., Cutrera, S., La Manna, M.P., Liberto, D.D., Vanini, V., Petruccioli, E., Dieli, F., Golletti, D., and Caccamo, N. (2015). Granzyme A as a potential biomarker of Mycobacterium tuberculosis infection and desease. Immunology Letters, 166:87-91.

7. Horwitz, M. A., Harth, G., Dillon, B. J., and Maslesa-Galic', S. (2000). Recombinant bacillus calmette-guerin (BCG) vaccines expressing the Mycobacterium tuberculosis 30-kDa major secretory protein induce greater protective immunity against tuberculosis than conventional BCG vaccines in a highly susceptible animal model. Proceedings of the National Academy of Sciences of the United States of America, 97(25): 13853-8.

8. Huang, C., Bi, E., Hu, Y., Deng, W., Tian, Z., Dong, C., Hu, Y and Sun, B. (2006). A novel NF-кB binding site controls human granzyme B gene transcription. Journal of Immunology, 176 (7) 4173-4181; DOI: https://doi.org/10.4049/jimmunol.176.7.4173

9. Huygen, K. (2014). The immunodominant T-cell epitopes of the mycolyl-transferases of the antigen 85 complex of M.tuberculosis. Frontiers in Immunology, 5:1-11. Doi: 10.3389/jimmu.2014.00321.

10. Invitrogen. (2010). ChampionTM pET SUMO Protein Expression System. User Manual; part no.25-0709.

11. Jiang, Q., Zhang, J., Chen, X., Xia, M., Lu, Y., Qiu, W., Feng, G., Zhao, D., Li, Y., He, F., Peng, G., and Wang, Y. (2013). A novel recombinant DNA vaccine encoding Mycobacterium tuberculosis ESAT-6 and FL protects against Mycobacterium challenge in mice. Journal of Biomedical Research, 27(5):406-420.

12. Jiang, Y., Liu, H., Li, M., Li, G., Pang, H., Dou, X., Zhao, X., and Wan, K. (2015). Single nucleotide polymorphism in Ag85 genes of Mycobacterium tuberculosis complex: Analysis of 178 clinical isolates from China and 13 BCG strains. International Journal of Medical Sciences, 12(2): 126-34. doi:10.7150/ijms.9951.

13. Kuo, C.J., Christopher, P., Ching, L., Bruce, L., and Yung, F. (2013). Elastin, a novel extracellular matrix protein adhering to mycobacterial antigen 85 complex. Journal of Biological Chemistry, 288 (6): 3886-3896.

14. Launois, P., Annie, D., Eliane, B., Pierre, C., Claire-Mich`ele, F., Jean-Paul, V., Kris, H. (2011). T cell reactivity against mycolyl transferase antigen 85 of $\mathrm{M}$. tuberculosis in HIV-TB coinfected subjects and in AIDS patients suffering from tuberculosis and nontuberculous mycobacterial infections. Clinical and Developmental Immunology. 10:1155-1166.

15. Li, W., Li, M., Deng, G., Zhao, L., and Wang Y. (2015). Prime-boost vaccination with bacillus calmette guerin and a recombinant aenoviru co-expressing CFP10, ESAT6, Ag85A and Ag85B of Mycobacterium tuberculosis induces robust antigen-specific immune responses in mice. Molecular Medicine Reports, 12:3073-3080.

16. Lu, C-C., Wu, T-S., Hsu, Y-J., Chang, C-J., Lin, C-S., Chia, J-H., Wu, T-L., Huang, T-T., Martel, J., Ojcius, D.M., Young, J.D., and Lai, H-C. (2015). NK cells killing mycobacteria directly by releasing perforine and granulysin. Journal of Leucocyte Biology, 96(6): 1119-1129.

17. McBryde, E.S., Meehan, M.T., Doan, T.N., Ragonnet, R., Marais, B.J., Guernier, V., and Trauer, J.M. (2017). The risk of global epidemic replacement with drug-resistant Mycobacterium tuberculosis strains. International Journal of Infectious Diseases, http://dx.doi.org/10.1016/j.ijid.2017.01.031.body.

18. Metcalfe, H.J., Steinbach, S., Jones, G.J., Connelley, T., Morrison, W.I., Vordermeier, M., Villarreal-Ramos, B. (2016). Protection associated with a TB vaccine is linked to increased frequency of Ag85A-specific CD+ T cells but no increase in avidity for Ag85A. Vaccine, 34: 4520-4525.

19. O'Shea, M.K., and McShane, H. (2016). A review of clinical models for the evaluation of human TB vaccine. Human Vaccine and Immunotherapeutics, 12(5): 1177-1187.

20. Otu, A.A. (2013). Is the directly observed therapy short course (DOTS) an effective strategy for tuberculosis control in a developing country. Asian Pacific Journal of Tropical Disease, 3(3): 227-231.

21. Piubelli, L., Campa, M., Temporini, C., Binda, E., Mangione, F., Amicosante, M., Pollegioni, L. (2013). Optimizing Escherichia coli as a protein expression platform to produce Mycobacterium tuberculosis immunogenic proteins. Microbial Cell Factories, 12:115.

22. Rizzi, C., Bianco, M.V., Blanco, F.C., Soria, M., Gravisaco, M.J., Montenegro, V., Vagnoni, L., Buddle, B., Garbaccio, S., Delgado, F., Leal, K.S., Cataldi, A.A., Dellagostin, O.A., and Bigi, F. (2012). Vaccination with a BCG strain overexpressing Ag85B protects cattle against Mycobacterium bovis challenge. PloS ONE, 7(12):e51396.

23. Silva, D., Ponte, C.G.G., Hacker, M.A., and Antas, P.R.Z. (2013). A whole assay as a simple, broad assessment of cytokines and chemokines to evaluate human immune responses to Mycobacterium tuberculosis antigens. Acta Tropica, 127:75-81

24. Tambunan, B.A., Priyanto, H., Nugraha, J., and Soedarsono. (2018). CD4+ and CD8+ T-Cells expressing interferon gamma in active pulmonary tuberculosis patients. African Journal of Infectious Disease, 12(S):49-53.

25. Tanghe, A., D’Souza, S., Rosseels, V., Denis, O., Ottenhoff, T.H.M., Dalemans, W., Wheeler, C., and Huygen, K. (2001). Improved immunogenicity and protective efficacy of a tuberculosis DNA vaccine encoding Ag85 by protein boosting. Infection and Immunity, 69(5):3041-3047.

26. Van-Crevel, R., Ottenhoff, T.H.M., and Vd Meer, J.W.M. (2002). Innate immunity to Mycobacterium tuberculosis. van 
Crevel, R., Ottenhoff, T.H.M., and van der Meer, J.W. (2002). Innate immunity to Mycobacterium tuberculosis. Clinical Microbiology Reviews, 15(2): 294-309.

27. Wang, X., Barnes, P.F., Dobos-Elder, K.M., Townsent, J.C., Chung, Y-T., Shams, H., Weis, S.E., and Samten, B. (2009). ESAT-6 inhibits of IFN- $\gamma$ by Mycobacterium tuberculosis-responsive human T-cells. Journal of Immunology. 182 (6): 3668-3677.

28. World Health Organization. (2016 ). Global Tuberculosis Control: WHO Report 2016. World Health Organization Press, Geneva, Switzerland.

29. Yuk, J-M., and Jo, E.-K. (2014). Host immune responses to mycobacterial antigens and their implications for the development of a vaccine to control tuberculosis. Clinical and Experimental Vaccine Research, 3(2):155-167. http://doi.org/10.7774/cevr.2014.3.2.155.

30. Zarif, R., Sankian, M., Gholubi, A., Farshadzadeh, Z., Soleimanpour, S., Youssefi, F., Varasteh, A.R. (2013). Cloning and expression of Mycobacterium tuberculosis major secreted protein antigen 85B (Ag85B) in Escherichia coli. Jundishapur Journal Microbiology, 6(2):112-116.

31. Zvi, A., Naomi, A., John, F., Jerald, C.S., and Avigdor, S. (2008). Whole genome identification of Mycobacterium tuberculosis vaccine candidates by comprehensive data mining and bioinformatics analyses. BMC Medical Genomics, 2008 1:18. https://doi.org/10.1186/1755-8794-1-18. 